\title{
Structural Design of Household OLED Modular Combination Lamp
}

\author{
Ruikun $\mathrm{Li}^{1, *}$, Xiaoming $\mathrm{Ji}^{2}$, Xupeng Wang ${ }^{3}$ \\ ${ }^{1}$ Department of Industrial Design, Xi'an University of Technology, Xi'an, 710054, China \\ 2 Department of Industrial Design, Xi'an University of Technology, Xi'an, 710054, China \\ ${ }^{3}$ Department of Industrial Design, Xi'an University of Technology, Xi'an, 710054, China
}

\begin{abstract}
With the improvement of people's life quality, the design of household lamps is not only limited to its function and practicability, but also has a higher exploration and pursuit in the expression of "home" culture in the shape of lamps.However modern lamps and lanterns is designed seldom according to the consistent that installs a style to come to put forward integral illume plan, it is difficult to satisfy human nature greatly, individuation, bedroom style diversity, and with a unity of modularity requirements.In this paper, the new lighting technology of OLED is studied in depth. Then, the method of modular design is applied to carry out innovative design of OLED household lighting fixtures, and a concrete design example is given.It not only enriches the design style of lamps and lanterns, but also pays attention to the independent demand of users' emotion.
\end{abstract}

\section{Introduction}

Lamps and lanterns design development so far into the concept and elements of many interesting, always bearing the humanities environment, lighting requirements, such as personalized formmedia is essential [1] .With the application of new materials, new processes and new technologies, the continuous reform and innovation of lighting technology have brought infinite possibilities to the design of lamps and lanterns, and they have also continuously designed lamps and lanterns with different emotional expressions for mainstream consumers. At present, modular design, as a highly practical design method, has been popular for many years and has been widely applied in various industries, effectively solving many thorny problems in design [2].Modular design thinking is applied to the design of lamps and lanterns are few and far between, how to meet the demand of personal aesthetic, and easy to form the overall design atmosphere, find truly meet the requirements of the modern market, the lamps and lanterns of consumers satisfied with the product, is the key point of this study.

\section{Development of OLED lighting technology}

OLED (Organic Light Emitting Diode) lighting technology is an innovative new planar lighting technology, which has been praised in the industry as the fourth revolution of the world lighting industry[3].Its flexible and curved features greatly improve the defects of LED technology, making it a new favorite in the lighting field [4].Its advantages are numerous and widely used, mainly reflected in the following 3 points :(1) the whole light source, the light is uniform, can achieve a large area; (2) Thin thickness, good flexibility and flexibility as shown in Figure 1; (3) Diverse light and color, arbitrary adjustment, personalized. Small to the family living flat lamp or commercial floodlight board, intelligent wear, big to the billboard of 200 square meters "wallpaper", etc.

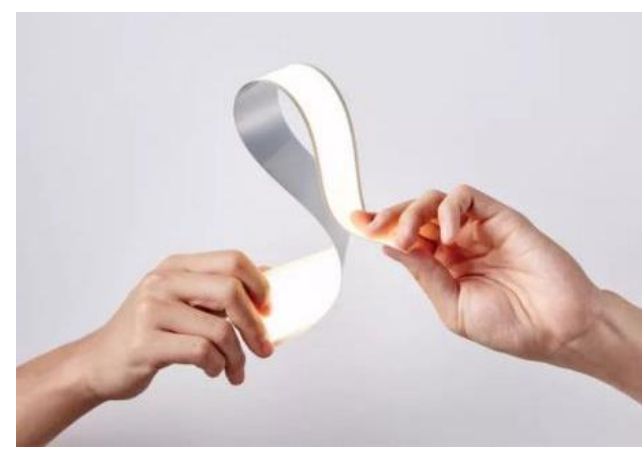

Fig. 1. OLED light belt

OLED technology was first discovered by Professor Deng Qingyun in an experiment by accident. At that time, there was no light source. After long-term research, Burroughes et al. [5] discovered OLED with luminous performance at the beginning of the 20th century, and since then, OLED luminous performance has been gradually applied to different fields.Abroad application of OLED light sources are mainly concentrated in the

*Corresponding author's e-mail: 1064068962@qq.com 
field of display, OLED materials has the characteristics of soft, good pervious to light, portable, many famous lighting companies such as GE, panasonic, philips, etc are devoted to OLED technology research and development and application, and the emergence of LG curved OLED TV shown in Figure 2 has greatly improved the traditional TV set. Samsung Galaxy Z Flip folding phone makes OLED curved technology more miniaturized and more mature in display technology.But technically foreign research focus mainly concentrated in OLED materials, light source, process, etc., but there are still problems of small volume and low luminous efficiency in OLED technology research [6], in the industrial distribution ways, there are now about 30 manufacturers into the luminescence materials research, and some large enterprises large lighting companies such as philips, GE, panasonic into OLED lighting technology the layout of the relevant patent and intellectual property research,it showed the tremendous potential of OLED lighting well with business opportunities.
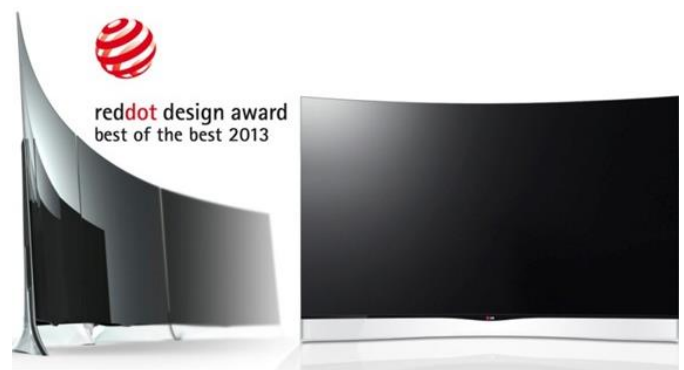

Fig. 2. LG surface splicing OLED TV

From the perspective of domestic development trend, the research on OLED lighting technology in China has made great progress, and China has become the second country with the large-scale production capacity of flexible OLED panels after South Korea.Most enterprises focus on improving the luminous efficiency, service life and production process of OLED lighting products [7].At present, using phosphorus as the main material of OLED greatly improves its efficiency, and has already surpassed the efficiency of incandescent lamp. In addition, in terms of lighting life, OLED is sufficient for portable equipment display screen, but for lighting, it needs to be improved twice to five times on the existing basis. Beijing Vicino company launched inch-level OLED display for the first time in 2000, which not only improved the luminous efficiency by $40 \%$, but also greatly improved the luminous life, setting off a research upsurge of OLED in China. However, China's research on OLED technology has never stopped. Figure 3 is huawei's upgraded folding phone Mate X, which won the public's favor once it was released. OLED, as the most important component of folding/curved phones, will be better and better developed and applied.

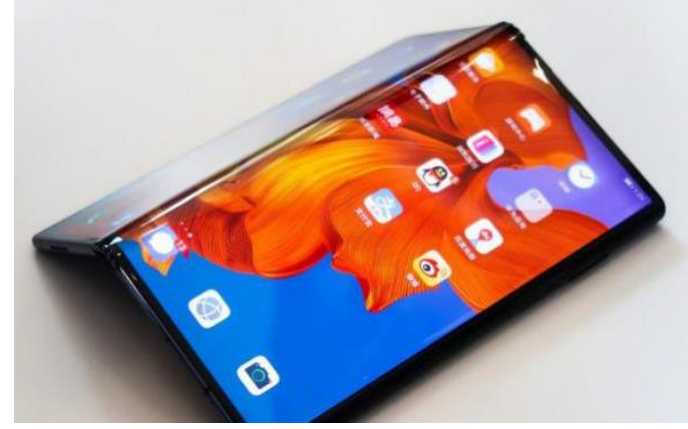

Fig. 3. Huawei upgraded folding phone Mate $X$

\section{Modularized lamp structure design}

Modularized design thinking has penetrated into every field of our life. In terms of lamp design, modularization breaks through the design principles of traditional lamps and lanterns, simplifies and standardizes the components of traditional lamps and lanterns, integrates light source, driver and components into a unified module, so that each part can be designed freely to generate new lamp styles, play different lighting purposes, realize the optimization of lighting effect, improve the practicability of lamps and shorten the design and development cycle and reduce the cost [8];Users can also design lamps and lanterns freely according to their own style and preferences. They only need to buy modules and then restructure some simple components to obtain unique and exquisite new lamps and lanterns at low price. Therefore, the idea of modular design plays an important role in the design of lamps and lanterns, which will be called the mainstream direction of the design of lamps and lanterns in the future.

\subsection{Introduction and development of the concept of modularity}

Modular structure design is the combination of certain factors in the process of product design, can form a certain function of the subsystems, as its name implies is with the help of a generic interface to edit each subsystem are combined,can produce different products [9], Solved the "one move affects the whole body" awkwardness.

Modular thinking was first applied to product design in 1900, a German company designed a modular bookshelf consisting of three different sizes of base, frame and roof. Users could choose different blocks and frames according to their preferences to combine their own bookshelves [10]. This kind of idea begins to be used extensively in household design at that time.

Modular design method is used to develop a lighting lamps and lanterns, can in the shortest possible time to meet changing market needs, reducing waste of raw materials to realize environmental protection and a multi-purpose content, and can greatly save the cost, through the reorganization of the components in the subsystem, the recycling and reuse can be achieved, which is conducive to reducing the environmental pressure and the adverse effects of artificial treatment, 
and fully meet the social requirements of economy and environmental protection.

\subsection{Basic features of modularity}

Modular design refers to that the whole system can be divided into several subsystems that can be combined with each other or act independently, and these subsystems have interfaces with the same structure and common functional units [11]. These subsystems are modules with the following characteristics:

(1) The module is independent. Modules have specific functions and are not limited by other modules, but exist independently. They will not change with the changes of other modules' functions. Because of its characteristics, the module can be used as a basic unit for independent production, processing and storage.

(2) The module is a component of the overall system. Modules constitute the system, and the system decomposes modules. Modules are replaceable, independent and composable, and can be combined to form a new system [12].

(3) The module is standard. The module has a common interface and a standardized usage feature. It can combine and innovate the interface of the product like lego.

(4) It has a unified corresponding interface. Modules can be combined into a required system through a common interface. Modules can share connections in order to achieve delivery and transport functions. The system becomes a new and ordered large system under the Mosaic combination of modules [13].

\subsection{Application of modularization in lamp design}

People's aesthetic tastes are constantly changing along with social factors, but the traditional products' forms and functions are too single to meet the different needs of consumers.Therefore, the modular division of the product and the formation of a variety of different styles and interesting products to meet the needs of the market is can't wait.

Modular unified ways can realize the individual needs of consumers, in the simplest way to satisfy their own independent design fun.The idea of modular design is applied in the design of lamps and lanterns. According to the change of consumers' aesthetic needs and the difference of the use environment, the general interface is used to replace the components of the chandelier, wall lamp, floor lamp, table lamp and so on to complete the design of a new overall system by themselves. Users can freely control the length, quantity and lighting scope of the lamps and lanterns [14], which not only saves resources but also easily forms the transformation between the old and the new product forms, satisfying the consumers' "playing heart" design, as shown in Figure 4.

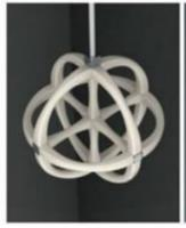

(a)droplight

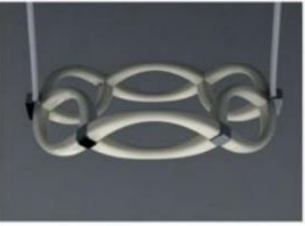

(b)Floor lamp
Fig. 4. Series lamps

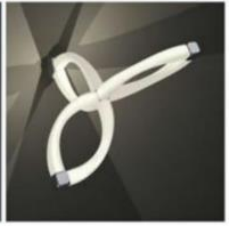

(c)Wall lamp

\section{Design and implement of lamps and lanterns}

On the basis of developing new material and technology of lamps, we should also consider how to satisfy the freshness and durability of the lamps[15]. Before lamps are designed, users' requirements for lamps should be fully understood, and modular design method should be adopted to combine and divide the sub-functions of lamps so as to realize users' personalized and customized demands.

\subsection{Preliminary design analysis}

(1)As far as the lamps and lanterns themselves are concerned, the vast majority of people match the lamps and lanterns for the bedroom when the house is decorated. Once the lamps and lanterns are selected well unless there is a problem, they will not be easily replaced which is undoubtedly very embarrassing for the consumers who pursue fashion and like new ideas. The modular series lamps and lanterns have a unified interface to realize the collocation of different modeling lamps and lanterns and meet the needs of consumers seeking novelty.

(2)According to the modular design principle, a completed lamp is composed of components with different sub-functions. As shown in Figure 5, The OLED surface light source and different components can be combined into lamps for different purposes through the standard interface connection, which can adds dynamic interest to the static lamps,in the process of personal assembly,it can also meet consumers' cognitive resonance and emotional integration towards lamps and lanterns[16].

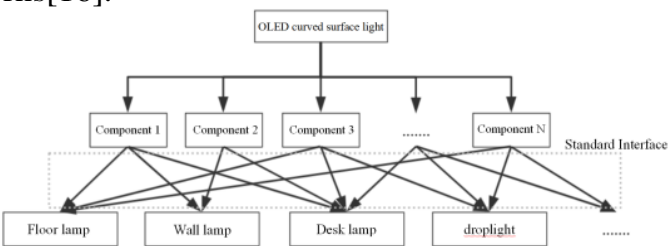

Fig. 5. Module component collocation

(3)The most direct advantage of adopting modular design is to simplify the overall design process, shorten the design and development cycle, reduce costs and at the same time meet the requirements of free combination design lamp modeling. The screw type and buckle type connection are adopted, the structure is simple, convenient and reliable, through the standard interface only need to change themodelling of OLED surface lamp, replace the type of components, to a certain extent 
greatly prolong the service life of lamps.Using the line surface body design combined into an organic whole,using a metal wire pipe with sufficient elasticity and rigidity, the port part is helical interface, the pipe body has invisible insert interface, the metal pipe is connected with the general helical structure infinitely, and the lamps and lanterns are assembled and matched freely, so as to realize the user's personalized design fun and complete multiple purposes from floor to suspension.

\subsection{User demand analysis and design positioning}

On the basis of users' cognition and experience of lamps and lanterns, the perceptual evaluation data of users on lamps and lanterns is collected through questionnaire survey. In combination with product design principles, user demand analysis data is obtained to determine the design positioning of lamps and lanterns.

(1)User demand analysis: In order to accurately grasp the development trend of modern lamps and lanterns design, we visited and surveyed various lamps and lanterns markets. The target group is the middle and low level consumer group between 25 and 40 years old. This kind of group is sensitive to fashion trend, lively, cognitive avant-garde, brave to try new things, prefer modern green and simple style, and has a certain emotional injection to the bedroom. The main consumption group is a modern society, on the choice of lamps and lanterns more practicality and uniqueness, give attention to two or morethings favored simple geometric shape on modelling, the material is still the first choice of metal and plastic on, metal good strength and toughness with plastic processing method is simple, low cost, good colour effect, choose contracted compact geometry and the metal line of variable length as modular elements, nimble diverse, rich cultural connotation, such the interactive design in people susceptible to emotional resonance.

(2)Design positioning: The increase of the living area of human promotes the diversification of lamps and lanterns. The modelling of lamps and lanterns depends on bedroom space area in very big part, can divide according to use environment roughly droplight, wall lamp, floor lamp, desk lamp.

As shown in Figure 6, the market research on lighting shows that: from the perspective of lighting source selection, most consumers are willing to try OLED lighting technology; in terms of style, simple modern style is more popular; From the material, the industrial process of plastic and metal collocation is still the mainstream;The mainly pure colors may be better.modern lamp design is not only a single combination of form, material and color,consider bedroom environment and human element synthetical however, undertake unified design with integral whole idea.

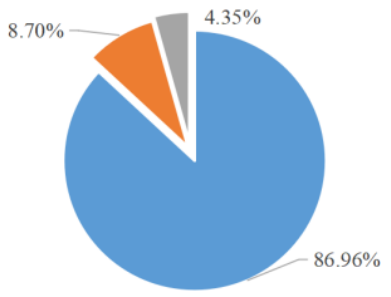

W. A. F'd love to = B. Unwilling and prefer to buy at will $\| \mathrm{C}$. other

(a)Whether is willing to Purchase a modular lamps and lanterns

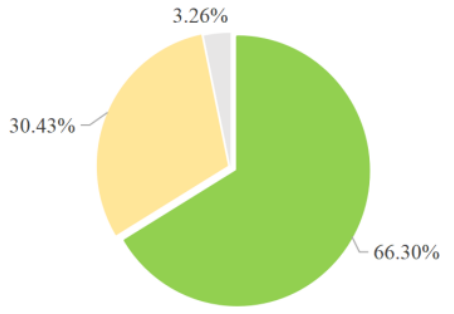

A.Yes, very interesting $\equiv$ B.No, too troublesome $=$ C. other

(b) Do like to assemble own lamps

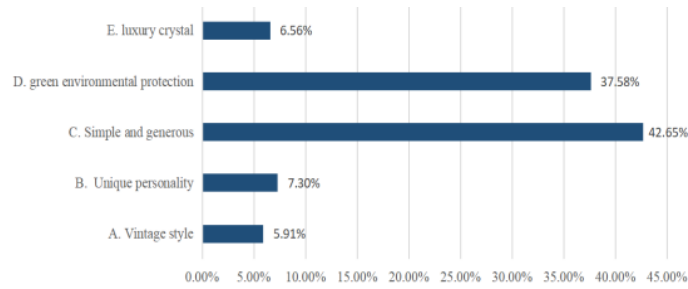

(c) Buy Lamps and lanterns style tendency

Fig. 6. Market research results

\subsection{Modular implementation plan}

Through the design of horizontal, vertical, linear and various types of combination ways of achieve the optimisatal lighting effect.Based on the flexible, thin and curved features of OLED surface lights, a group of panel lights are designed with relatively simple structure. As shown in figure 7 , module components mainly include OLED surface lights (of various sizes), buckle joints, and metal wire tube. The biggest advantage of embedding metal buckle as a standard interface in wood is that wood has good insulation and is easy to be processed, while spiral splicings of metal wire tube directly eliminate the traditional lamp pole and base structure, and can be modified at will.

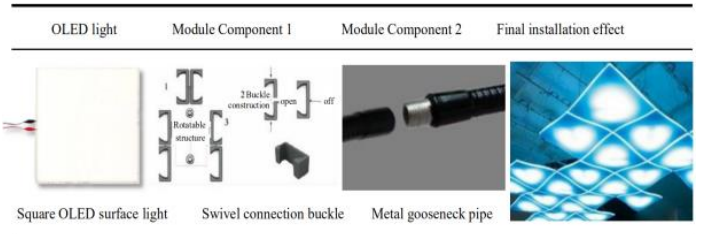

Fig. 7. Design process of selected module component combination lamps 


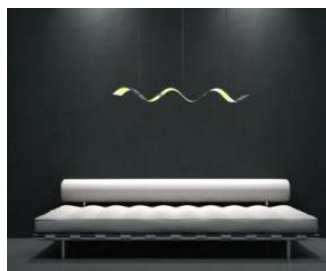

(a) Module combination lights

A block of flat panel lights of several by the same square thin OLED panel hooking each other in figure 8 (a), the sub-panel is inlaid by OLED surface light source and fixed in a metal sheet of equal size with a certain volume,can be flexible changed straight and softened.In addition, the four panels can be used independently. When put together, they can be used as a large chandelier. Independently, they can be freely folded and curled into their favorite shape(figure 8 (b)).Especially when it is droplight of various wave curve is very dynamic, light is refracted through OLED surface light scattering through metal into a wall, the light becomes more soft can be used as a good wall lamp, of course,put on the table to save the space taken up by the bracket, such a surface light in production and transportation more space saving more convenient.

OLED surface light can have countless folded modelling, also can have several changes shapes, based on the advantage of OLED curved surface light source, using a standard interface to edit modular parts , as well as deformable metal of wire pipe can greatly meet the different demand of the bedroom.It break through the traditional design of lamps and lanterns can't satisfy the lamps and lanterns of follow one's inclinations forms and structure change of bottleneck, fully stimulate the practice pleasure and emotional needs of users, implements the green development of the modern design of lamps and lanterns.

\section{Conclusion}

In this paper,based on the flexibility and the thickness of the small advantage of new type surface OLED lighting technology, using the modular design thinking, will contracted compact geometry and the metal line of variable length as modular elements for design of lamps and lanterns,conform to the aesthetic needs of contemporary and contracted but not simple, also make the bedroom of the modelling of lamps and lanterns in different characteristics at the same time, but one integrated.Its fun operation provides fun for home life, which not only realizes the diversified form of lamps and lanterns, but also meets the green and environmental protection concept of reuse.For household lighting design to achieve the "new" and "change" personalized display, contribute to the advancement of design and innovation of lamps and lanterns.

\section{Reference}

1. Fu Zhichao. On the Development Trend of Modern Lamp Modeling Design [J]. Big Stage, 2013(7):164-165.

2. Chen Tao. Research on modular Design of Outdoor Lamps [D]. Nanjing University of Science and Technology, 2015.

3. Liu Fei, LI Xiao, FENG Xiaohua. Progress in OLED lighting Technology and Its Application [J]. Journal of Lighting Engineering, 2014(3):93-97.

4. Wang Wenjing. Application research of OLED technology in lighting Product Modeling design [D]. East China University of Science and Technology, 2013.

5. Di S, Guan X, Xiao W. Design and realization of an OLED application with DC-DC converter[C]// International Conference on Consumer Electronics, Communications and Networks. IEEE, 2012:2498-2500.

6. Singh R, Unni K N N, Solanki A, et al. Improving the contrast ratio of OLED displays: An analysis of various techniques[J]. Optical Materials, 2012, 34(4):716-723.

7. He Yisheng. Development Trend and Characteristics of Lamps and Lanterns at home and abroad [J]. Lamp \& Lighting, 2000, 24(2):16-17.

8. Peng Kexing, ZHANG Liwei. Development and Design of LED Indoor Lighting Series based on Modularization [J]. Machinery Design, 2013, 30(11):108-111.

9. Jin Xinmin, GAO Qimin. Research on modular Lamp Design [J]. Art \& Technology, 2013, 26(5):167-168.

10. Shao Shengnan. Application Research of Modular Concept in Bookshelf Design [D]. Hebei University of Science and Technology,2018.

11. Hou liang, xu yanshen, li sen et al. Generalized modular design module coding system for mechanical products based on template modules. Mechanical design,2002,19(1):8 10.

12. Zhou linyan. Modular design strategy for mechanical products $[\mathrm{J}]$. Southern agricultural machinery,2020,51(01):144.

13. Li Hao, TAO Fei, Wen Xiaoyu, Wang Haoqi, Luo Guofu. Modular Design for Large-scale personalized Product Service System [J]. China Mechanical Engineering, 2012,29(18):2204-2214+2249.

14. Jia Jingjing. Application of mortise and tenon joint structure in luminaire design $[\mathrm{J} / \mathrm{OL}]$. Packaging Engineering :1-8[2020-07-30].

15. Cheng L R. Principles and Procedures of Lamp Design $[\mathrm{J}]$. Science and Technology Information, 2016, 14(31):164-165.

16. Chi Ruifeng, JIANG Jia-xiu. Research on Emotional Design of Modern Lamps [J]. Packaging Engineering, 2013(24):59-62. 